19

\title{
Дисперсия поверхностных плазмонов в структурах с проводящей пленкой
}

\author{
(C) М.В. Давидович
}

Саратовский государственный национальный исследовательский университет им. Н.Г. Чернышевского, 410012 Саратов, Россия

e-mail: DavidovichMV@info.sgu.ru

Поступила в редакцию 01.08.2018 г.

В окончательной редакции 22.11.2018 г.

Принята к публикации 04.12.2018 г.

Исследованы дисперсионные уравнения, замедление, групповая скорость, условия для прямых и обратных, медленных и быстрых плазмонов вдоль тонких металлических пленок, а также графеновых листов, в том числе расположенных на диэлектрическом слое и в диэлектрике. Рассмотрены недиссипативные и диссипативные поверхностные плазмоны. Обратный недиссипативный симметричный плазмон не наблюдается в диссипативной пленке серебра. Показано, что из аномальной отрицательной дисперсии диссипативного плазмона не следует, что он обратный. Для обратного диссипативного плазмона входной импеданс структуры со стороны вакуума должен быть емкостным, а плазмон втекающим. Рассмотрены симметричные и несимметричные структуры с такими свойствами.

DOI: $10.21883 /$ OS.2019.03.47379.228-18

\section{Введение}

В тонких металлических пленках с толщиной $t$ порядка десятков нанометров без диссипации возможны аномальная отрицательная дисперсия и обратные симметричные поверхностные плазмоны (ПП) [1-11]. Для них движение энергии в слое из-за отрицательного значения диэлектрической проницаемости (ДП) противоположно и превалирует над ее движением в окружающем пространстве [2]. Учет диссипации в таких структурах выполнен в работах [6-11]. В последнее время исследуется ПП вдоль листов графена, бислоя графена, листов графена на диэлектрической подложке, графеновых нанолент [12-23]. В таких структурах также возможны прямые и обратные ПП. Диссипация приводит к сложному характеру дисперсионных кривых, при этом в областях максимального замедления потери велики даже при малой диссипации в среде, а тип дисперсии (нормальная или аномальная отрицательная) уже не определяет тип волн (прямые они или обратные) $[6,8,11]$. Для выяснения указанного вопроса следует вычислять проинтегрированный по сечению вектор Пойнтинга $\mathbf{S}=\operatorname{Re}\left(\mathbf{E} \times \mathbf{H}^{*}\right) / 2$ или скалярное произведение $\mathbf{k}^{\prime} \cdot \mathbf{k}^{\prime \prime}$ реальной и отрицательной мнимой частей комплексного волнового вектора $\mathbf{k}^{\prime}=\mathbf{k}^{\prime}-i \mathbf{k}^{\prime \prime}$ [6-8]. Далее везде штрих означает реальную часть, а двойной штрих отрицательную мнимую часть. Обратному диссипативному плазмону соответствует отрицательное значение, $\mathbf{k}^{\prime} \cdot \mathbf{k}^{\prime \prime}<0$ (затухание движется противоположно фазе), что эквивалентно условию $\mathbf{k}^{\prime} \cdot \mathbf{S}<0$. Вместо групповой скорости (ГС) следует использовать скорость энергии $\mathbf{v}_{c}=\mathbf{S} /\langle w\rangle$, где средняя плотность энергии за период для однокомпонентной холодной плазмы известна:

$$
\langle w\rangle=\frac{\varepsilon_{0}\left|\mathbf{E}^{2}\right|}{4}\left(\varepsilon_{L}+1+\frac{\omega_{p}^{2}\left(\omega^{2}+\omega_{c}^{2}\right)}{\left(\omega^{2}-\omega_{c}^{2}\right)^{2}+\omega^{2} \omega_{c}^{2}}\right) .
$$

Мы полагаем, что ДП металлической пленки в этом случае описывается формулой Друде-Лоренца

$$
\varepsilon(\omega)=\varepsilon_{L}-\omega_{p}^{2} /\left(\omega^{2}-i \omega \omega_{c}\right)
$$

Величина $\varepsilon_{L}$ в рассматриваемом диапазоне считается вещественной и не зависящей от частоты. Она определяет ДП кристаллической решетки и вклад в ДП от межзонных переходов, а ее частотная зависимость (дисперсия) проявляется на более высоких частотах. Отсутствию диссипации соответствует нулевая частота столкновений $\omega_{c}$. Отсутствию плазмы носителей соответствуют их нулевые концентрация и плазменная частота $\omega_{p}=0$. Тогда $\langle\omega\rangle=\varepsilon_{0}|\mathbf{E}|^{2}\left(\varepsilon_{L}+1\right) / 4$ есть плотность энергии в недиссипативном диэлектрике с ДП $\varepsilon_{L}$. В диссипативном диэлектрике следует использовать $\varepsilon_{L}^{\prime}=\operatorname{Re}\left(\varepsilon_{L}\right)$ [24]. Частота поверхностного плазмонного резонанса (ППР) $\omega_{s p}=\sqrt{\omega_{p}^{2} /\left(\varepsilon_{L}+1\right)-\omega_{c}^{2}}$ соответствует условию $\varepsilon^{\prime}=-1$, и при малой диссипации в $\sqrt{\varepsilon_{L}+1}$ раз (т.е. в несколько раз) меньше плазменной частоты [25-28].

Цель настоящей работы - выяснение хода дисперсионных кривых ПП в тонких пленках и структурах с тонкими пленками без диссипации и с диссипацией и также исследование характера и типа диссипативных ПП. 


\section{Дисперсия ПП для одиночной проводящей пленки}

В статьях [1-4] и монографиях [25-28] дисперсия ПП для одиночной пленки приведена достаточно схематично, так что дисперсионная ветвь симметричного электрического ПП (Е-ПП) стремится к частоте ППР $\omega_{s p}$ сверху, а антисимметричного - снизу. В очень толстой пленке (когда ПП на обеих ее сторонах не взаимодействуют) обе дисперсионные ветви сливаются и подходят к частоте ППР снизу. Ясно, что оба случая реализованы быть не могут. Далее показано, что во всех случаях ветви подходят к $\omega_{s p}$ снизу, но для симметричного Е-ПП имеют место два перехода через частоту $\omega_{s p}$ при двух значениях компоненты волнового вектора, так что условие $-1<\varepsilon^{\prime}<0$ реализуется в конечной области $k_{x 1}<k_{x}<k_{x 2}$ значений компоненты $k_{x}$ волнового вектора. С ростом толщины эта область сдвигается к бесконечности, что делает область $-1<\varepsilon^{\prime}<0$ запрещенной. Диссипация ограничивает замедление и делает возможным распространение сильно затухающих ПП в запрещенной зоне. Картины диссипативных дисперсионных ветвей для ПП Ценнека имеются в ряде руководств по плазмонике [26-28]. Стремление ветви сверху еще невозможно и потому, что в диссипативном случае существует ветвь с отрицательной дисперсией, которая уже подходит к частоте ППР (и к точке максимального замедления) сверху.

Сначала рассмотрим простую задачу определения дисперсионных ветвей простейших пленочных структур без диссипации. Будем использовать волновое число $k_{0}=\omega / c$, плазменное волновое число $k_{p}=\omega_{p} / c$ и плазменную длину волны $\lambda_{p}=2 \pi / k_{p}$. Для серебра $\lambda_{p} \approx 100 \mathrm{~nm}, \varepsilon_{L} \approx 10$. В соответствии с этим частоту будем связывать с ДП: $k_{0}=k_{p} / \sqrt{\varepsilon_{L}-\varepsilon}$. Для четного (симметричного) Е-ПП, идущего вдоль оси $x$, имеет место дисперсионное уравнение (ДУ) [6,7]

$$
n=\frac{k_{x}}{k_{0}}=\sqrt{\frac{\varepsilon^{2}-\varepsilon \tanh ^{2}(\theta)}{\varepsilon^{2}-\tanh ^{2}(\theta)},}
$$

где $\theta=t \sqrt{k_{x}^{2}-k_{0}^{2} \varepsilon} / 2=\left(t k_{0} / 2\right) \sqrt{n^{2}-\varepsilon}$. Поле такого Е-ПП определяется при электрической стенке в центре пленки $(z=0)$, т.е. при нечетной компоненте $E_{x}(z)$ и четных поперечных компонентах $E_{z}(z)$ и $H_{y}(z)$. Для Е-ПП с магнитной стенкой ДУ получается из (1) заменой $\tanh ^{2}(\theta) \rightarrow \tanh ^{-2}(\theta)$. В литературе приняты названия соответственно симметричный и антисимметричный Е-ПП. Такие ПП возбуждаются падающей плоской волной с $p$-поляризацией. Магнитный Н-ПП с магнитной стенкой имеет ДУ, которое получается из (1) заменой $\varepsilon^{2} \rightarrow 1$. Если в этом ДУ затем сделать замену $\tanh ^{2}(\theta) \rightarrow \tanh ^{-2}(\theta)$, то получим ДУ магнитного Н-ПП с электрической стенкой [6,7]. Однако такой Н-ПП диссипативный, вытекающий даже если в пленке диссипации нет. Все перечисленные кроме него ПП недиссипативные при $\varepsilon<0$ (т.е. при $\omega<\omega_{p}$ ). Н-ПП возбуждается волной с $s$-поляризацией.

Е-ПП (1) интересен тем, что может быть обратным. Несмотря на простоту ДУ (1), оно в литературе до конца не проанализировано. Цель работы - подробное исследование ДУ (1), влияние диссипации на его решение и на решения других простейших ДУ для тонких проводящих пленок на диэлектрической подложке. Исследование недиссипативных решений необходимо для поиска комплексных корней в присутствии диссипации, когда ДП комплексная. При диссипации частота $\omega_{s p}=\omega_{p} / \sqrt{\varepsilon_{L}+1}$ смещается вниз. Бесконечно малая диссипация при большой толщине $(t \rightarrow \infty)$ приводит к предельному значению $\tanh (\theta) \rightarrow 1$ ДУ (1) и ДУ нечетного (несимметричного) Е-ПП вырождаются в уравнение Ценнека $n=\sqrt{\varepsilon /(\varepsilon+1)}$. Оно явное и имеет решение при $\varepsilon<-1$ в виде медленного Е-ПП и при $\varepsilon>0$ в виде быстрого Е-ПП. В запрещенной зоне $0<\varepsilon<-1$ плазмонные волны возможны только при диссипации, при этом возникающие отрицательные аномальная дисперсия и (ГС) $v_{g}$ соответствуют прямому медленному и быстрому диссипативному ПП [6-10]. Без диссипации в запрещенных зонах имеются чисто мнимые (т.е. затухающие) решения. Кроме обратных Е-ПП (1) возможны обратные Н-ПП с ДУ

$$
n=\sqrt{\left(1-\varepsilon \tanh ^{2}(\theta)\right) /\left(1-\tanh ^{2}(\theta)\right)} .
$$

Однако они очень медленные в низкочастотной области и при уменьшении частоты, когда пренебречь частотой столкновений $\omega_{c}$ уже нельзя, диссипативные. Существенно понизить $\omega_{c}$ можно при сверхнизких температурах. Кроме того, такие ПП не возбуждаются продольными электронными пучками и волнами с $p$ поляризацией.

При решении (1) в области $-\infty<\varepsilon<-1$ с фиксированной ДП возможно наличие либо одного, либо двух, либо трех корней. При $\varepsilon=-1$, как видно, имеет место корень $n=\infty$. Кроме него имеет место еще два корня, положение которых определяется толщиной $t$. Уравнение для соответствующих замедлений имеет вид

$$
n^{2}=\left\lfloor 1+\tanh ^{2}\left(\alpha \sqrt{n^{2}+1}\right)\right\rfloor /\left\lfloor 1-\tanh ^{2}\left(\alpha \sqrt{n^{2}+1}\right)\right\rfloor .
$$

Число корней и их положение зависят от параметра $\alpha=k_{0} t / 2$. Если ищется не очень большой корень $n$ и $\alpha \sqrt{n^{2}+1} \ll 1$, то

$$
n^{2}=\left\lfloor 1+\alpha^{2}\left(n^{2}+1\right)\right\rfloor /\left\lfloor 1-\alpha^{2}\left(n^{2}+1\right)\right\rfloor
$$

и

$$
n^{2}=\left(1+1 / \alpha^{2}\right) /\left(1 / \alpha^{2}-2\right) \approx 1+3 \alpha^{3} .
$$

Если ищется большой по значению корень, то

$$
\tanh ^{2}\left(\alpha \sqrt{n^{2}+1}\right) \approx 1-4 \exp \left(-2 \alpha \sqrt{n^{2}+1}\right) .
$$

Тогда $n^{2}=\exp \left(2 \alpha \sqrt{n^{2}+1}\right) / 2-1 . \quad$ При $\quad-1<\varepsilon<$ $<\varepsilon_{m}<0$ имеет место либо два корня, либо один корень 
при максимальном значении $\varepsilon=\varepsilon_{m}$, либо корней вообще нет. Это иллюстрирует рис. 1, где показан численно полученный ход кривых дисперсионных ветвей. Из него видно, что область аномальной дисперсии, где имеет место отрицательная ГС и обратный ПП, содержит в себе точку $\omega_{s p}$. Для нахождения крайних частот следует решать уравнение $v_{g} / c=\partial k_{0} / \partial k_{x}=0$. Для этого дифференцируем ДУ (1), полагая $v_{g=0}$ :

$$
1=k_{0} \frac{\partial}{\partial k_{x}} \sqrt{\left(\varepsilon^{2}-\varepsilon \tanh ^{2}(\theta)\right)} .
$$

В результате имеем

$$
1=\frac{\varepsilon(1-\varepsilon) k_{x} k_{0} t \tanh ^{3}(\theta)\left(1-\tanh ^{2}(\theta)\right)}{2 \sqrt{k_{x}^{2}-k_{0}^{2} \varepsilon}\left(\varepsilon^{2}-\tanh ^{2}(\theta)\right)^{3 / 2} \sqrt{\left(\varepsilon^{2}-\varepsilon \tanh ^{2}(\theta)\right)}}
$$

Выражая из (1) $\tanh ^{2}(\theta)=\varepsilon^{2}\left(n^{2}-1\right) /\left(n^{2}-\varepsilon\right)$, получим условие для обратного ПП:

$$
1=k_{0} t\left(n^{2}-1\right)^{3 / 2}(1+\varepsilon) / 2 .
$$

Учитывая связь $k_{0}=k_{p} / \sqrt{\varepsilon_{L}-\varepsilon}$, из (3) получаем

$$
\begin{aligned}
\varepsilon= & -\left(1+\frac{2}{k_{p}^{2} t^{2}\left(n^{2}-1\right)^{3}}\right) \\
& \pm \sqrt{\left(1 \frac{2}{k_{p}^{2} t^{2}\left(n^{2}-1\right)^{3}}\right)^{2}+\frac{4 \varepsilon_{L}}{k_{p}^{2} t^{2}\left(n^{2}-1\right)^{3}}-1 .}
\end{aligned}
$$

Подстановка (4) в (1) дает трансцендентное уравнение относительно замедлений, на которых ГС обращается в ноль. Указанные корни следует находить численно.

Численные расчеты показывают, что для толстой пленки область аномальной отрицательной дисперсии прижата к частоте $\omega_{p s}$, где $\varepsilon=-1$. Для очень тонкой пленки имеется резкий и узкий пик, а далее эта область опять прижата к частоте плазмонного резонанса. Так, максимум кривой $2(t=50 \mathrm{~nm})$ имеет место при $\varepsilon=-0.75$, а в области минимума $\varepsilon=-1.012$. За ней при увеличении замедления ГС возрастает от нуля до небольшого максимума и затем начинает убывать до нуля при $n \rightarrow-\infty$. С уменьшением толщины максимум уменьшается и смещается влево, как и минимум. С сильным увеличением толщины происходит большое смещение максимума вправо в область больших замедлений, при этом ДП для максимума $\varepsilon \approx-1$ (чуть превышает это значение). ДП минимума лежит чуть ниже $\varepsilon=-1$. Такие образом, при максимальном замедлении все кривые подходят к области ППР по частоте снизу, а не сверху, как это графически представлено в ряде работ [1-4]. Это объясняется тем, что стремление $\tanh (\theta) \rightarrow 1$ существенно (экспоненциально) быстрее, чем стремление $\varepsilon \rightarrow-1$, поэтому без диссипации при бесконечном замедлении $\varepsilon=-1-0$, а решения в области $\varepsilon=-1+0$ нет. В области прижатия дисперсионной кривой к области $\varepsilon=-1$ и больших замедлений отрицательная ГС весьма мала по модулю, что говорит о почти

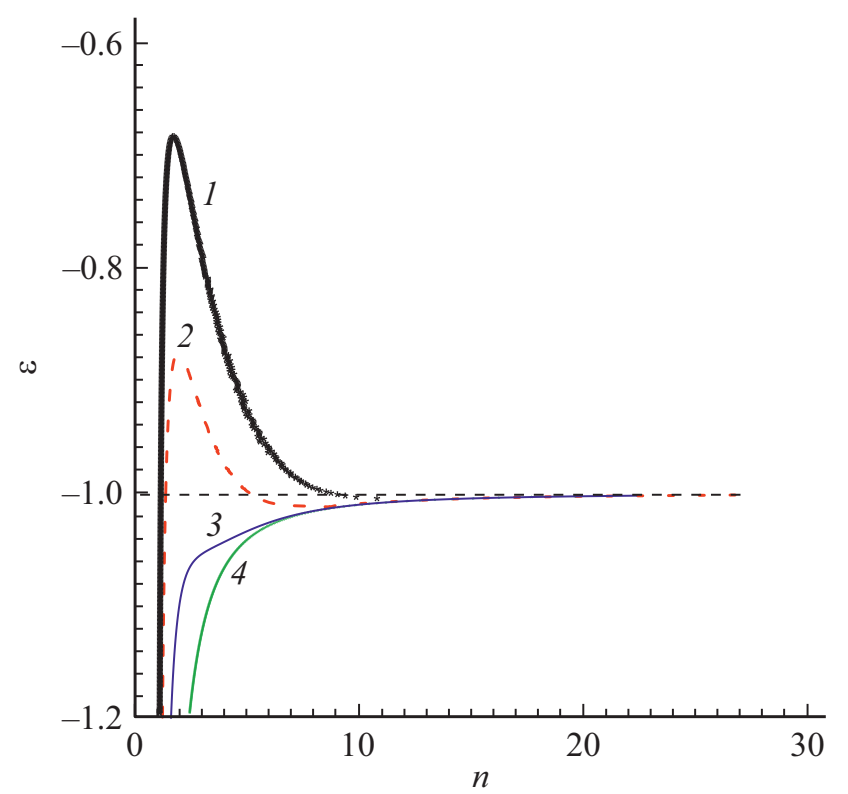

Рис. 1. Диэлектрическая проницаемость в зависимости от замедления недиссипативного симметричного Е-ПП вдоль серебряной пленки $\left(\omega_{p}=1.9 \cdot 10^{16}, \varepsilon_{L}=10\right)$ с толщинами $t=40$ (кривая 1), 50 (2), 70 (3), $500 \mathrm{~nm}(4)$.

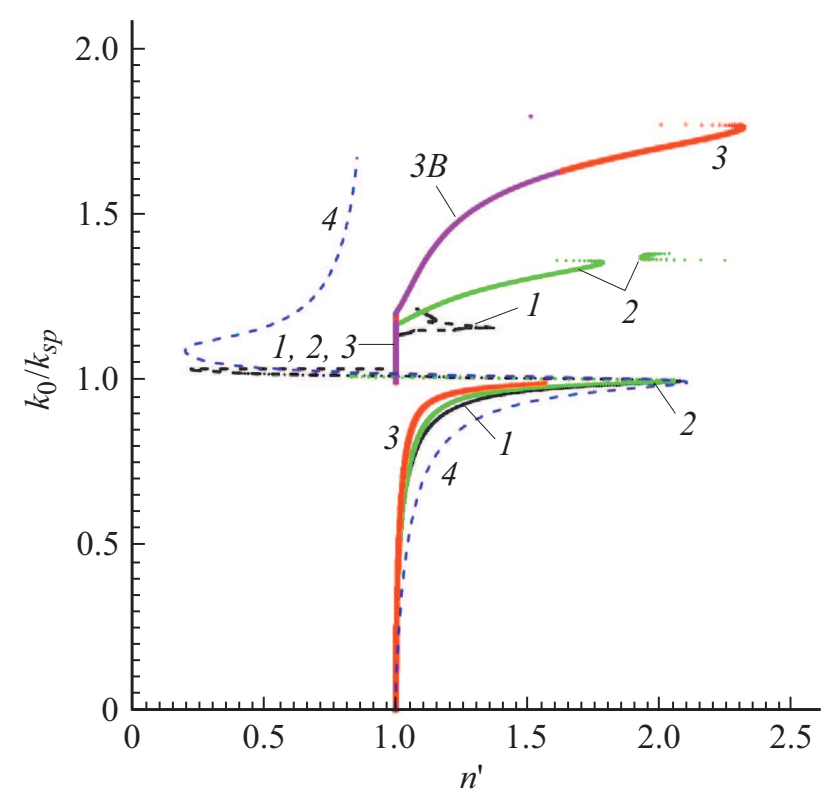

Рис. 2. Нормированное к $k_{s p}=c^{-1} \sqrt{\omega_{p}^{2} /\left(\varepsilon_{L}+1\right)-\omega_{c}^{2}}$ волновое число в зависимости от замедления $n^{\prime}$ симметричного плазмона в пленке серебра $\left(\omega_{p}=1.9 \cdot 10^{16}, \omega_{c}=5 \cdot 10^{13} \mathrm{~Hz}\right.$, $\left.\varepsilon_{L}=10\right)$ с толщинами $t=50$ (кривая 1), 20 (2), $10 \mathrm{~nm}$ (3), $\infty(4)$.

резонансном волновом процессе. Несколько увеличить модуль ГС можно для наноразмерных пленок при не слишком больших замедлениях. Это очень узкая область для $k_{x}$.

Диссипация приводит к ограничению максимального замедления. Итерационное решение ДУ (1) для сереб- 


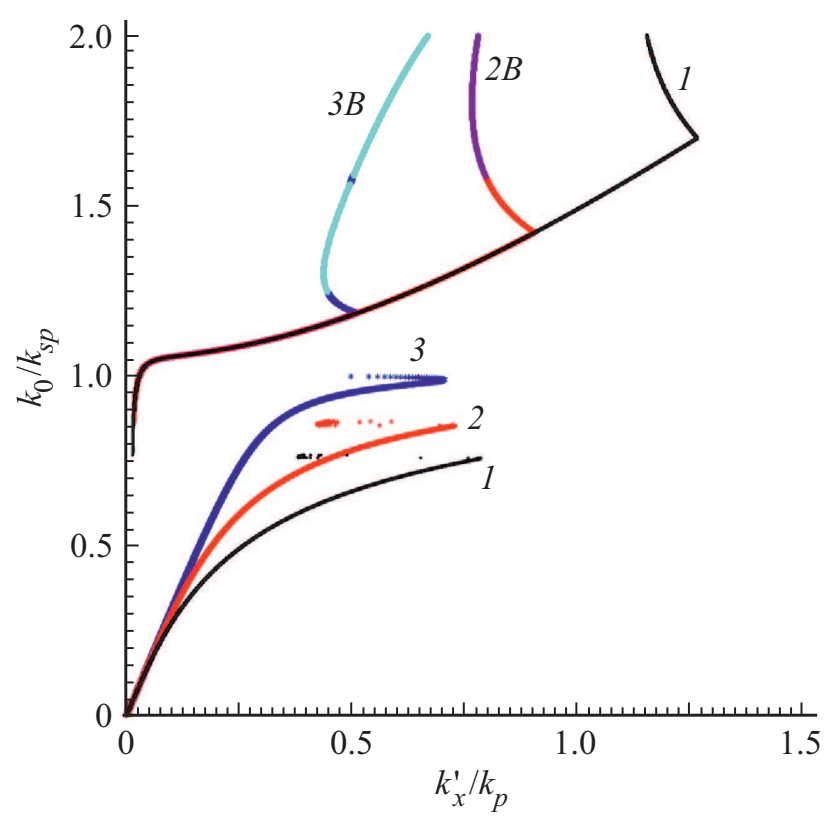

Рис. 3. Нормированная к плазменному волновому числу дисперсия антисимметричного (с магнитной стенкой) Е-ПП в слое серебра с толщинами 5 (кривая 1), 10 (2), $50 \mathrm{~nm}$ (3). Символами $B$ обозначены соответствующие части кривых, где ПП обратные.

ряной пленки $\left(\omega_{c}=5 \cdot 10^{13} \mathrm{~Hz}\right)$ приведено на рис. 2 , а для несимметричного ПП - на рис. 3. На графики выведены точки решения ДУ, для которых невязки были менее $10^{-5}$. Для получения большего количества точек в области резонанса необходим очень маленький шаг по частоте. Все кривые подходят к области максимального замедления снизу. Если без диссипации переход от нормальной дисперсии к аномальной отрицательный (и наоборот) происходит в точке, где $v_{g}=0$, то в диссипативном случае ГС сначала сильно уменьшается при подходе к точке $k_{s p}$, но нуля не достигает. Затем она начинает резко возрастать, переходит через бесконечность и меняет знак [6-10].

Несимметричный (с магнитной стенкой) ПП более медленный. Его максимальное замедление увеличивается с уменьшением толщины. В области максимального замедления потери ПП также максимальны, причем они растут с уменьшением $\omega_{c}$ (т.е. с уменьшением диссипации). Для ПП Ценнека вдоль полупространства $(t=\infty$, кривая 4 , рис. 2) решение аналитическое, и для максимального замедления имеем [10]

$$
n_{\max }^{\prime}=\left(1+\varepsilon^{\prime \prime} / 2\right) / \sqrt{2 \varepsilon^{\prime \prime}}, \quad n_{\max }^{\prime \prime}=\left(1+\varepsilon^{\prime \prime} / 2\right) / \sqrt{2 \varepsilon^{\prime \prime}} .
$$

Максимальное замедление достигается при $\varepsilon^{\prime}=-1$. Если диссипация очень малая, то $n_{\max }^{\prime} \approx n_{\max }^{\prime \prime}$, т.е. замедление и потери ПП очень велики. Вычисления диссипации и вектора Пойнтинга показывают, что в области аномальной отрицательной дисперсии ПП прямой. Обратный замедленный ПП возникает для кривой 3 (область $3 B$ ). Он идентифицирован по вычисленным отрицательным потерям (по отношению к положительной фазовой постоянной $k_{x}^{\prime}$ ). Эта область соответствует $\varepsilon^{\prime}>1$, что для серебра достигается в ультрафиолете.

Рассмотрим вопрос, почему возникает отрицательная дисперсия. Для ПП (1) плотность тока поляризации $J_{x}(z)=i \omega \varepsilon_{0}(\varepsilon-1) E_{x}(z)$ поддерживает ПП, поле которого ее и возбуждает. В силу нечетности $E_{x}(z)$, интегрируя $J_{x}(z)$ в областях $(0, t / 2)$ и $(-t / 2,0)$, получим две поверхностные плотности тока на обеих поверхностях пленки, $J_{s}^{+}=-J_{s}^{-}$. Поле таких двух волн поверхностного тока дается формулами (2.41) работы [29]. Если толщина мала, эти токи частично компенсируют возбуждаемые в вакууме поля, и поток энергии в пленке может превысить поток в вакууме. При увеличении толщины указанный эффект наступает при больших замедлениях, когда поле более сильно прижато к поверхностям. При этом превышение небольшое, и ГС мала. Увеличение толщины пленки приводит к увеличению потока мощности в вакууме, и выполнение условия $\left\langle S_{x}\right\rangle=0$ становится возможно при существенно больших замедлениях, когда трудно различить отклонение дисперсионной кривой от прямой $\varepsilon=-1$. При очень большой толщине металлической пластины поля по обе ее стороны не связаны в силу экспоненциального затухания внутри пленки. При бесконечной толщине эффект не наблюдается. В этом случае энергия в металлической полуплоскости движется в тонком ее слое, определяемом глубиной проникновения, и ее поток не может превысить поток в вакууме, определяемый током в указанном слое.

В недиссипативном случае при бесконечном замедлении на частоте $\omega_{s p}$ ГС обращается в нуль, но не меняет знак. Компонента вектора Пойнтинга $S_{x}$ в вакууме имеет знак $k_{x}$. Знак $S_{x}$ в пленке определяется величиной $\varepsilon k_{x}$. Знак компоненты $\left\langle S_{x}\right\rangle$, где скобки Дирака означают интеграл в пределах $(-\infty, \infty)$, определяется разностью потоков. Условие $\left\langle S_{x}\right\rangle=0$ дает альтернативный к (2) способ определения точек области аномальной отрицательной дисперсии. Диссипация приводит к конечному максимальному замедлению, при этом и потери ПП максимальные, а компонента $\left\langle S_{x}\right\rangle$ зависит от $k_{x}^{\prime}$ и $k_{x}^{\prime \prime}$. Смена знака ГС становится возможной в точке максимального замедления путем перехода через бесконечность [6$10,20]$, что еще раз подтверждает необходимость использовать $\left\langle S_{x}\right\rangle$ для классификации ПП.

\section{Дисперсия в простейших структурах с проводящей пленкой}

Рассматривать тонкую металлическую пленку реально либо внутри весьма толстого диэлектрика с ДП $\tilde{\varepsilon}$, либо на диэлектрической подложке. В первом случае ДУ (1) следует модифицировать путем замены $k_{0} \rightarrow k_{0} \sqrt{\tilde{\varepsilon}}$. Во втором случае подложки толщины $d$ и пленки толщины $t$ ДУ приведены в работах $[4,6-10]$. Вид ДУ зависит от типа ПП. Вне диссипативной структуры следует искать 
поверхностные ПП с экспоненциальным убыванием поля на бесконечности. Однако с понижением частоты медленный ПП может смениться на быстрый, вытекающий, антиповерхностный с экспоненциальным нарастанием поля на бесконечности $[10,30]$. В диссипативной структуре возможно втекание волны из бесконечности в нее, а также и вытекание. Получить ДУ можно путем сшивания решения волновых уравнений в подобластях, путем использования матриц передачи слоев (их перемножение и приводит к сшиванию), методом трансформации импедансов и еще рядом способов [6-9], в том числе с использованием функций Грина и интегральных уравнений (ИУ) $[8,9]$. ИУ позволяют описывать дисперсию в неоднородных пленках и структурах. В несимметричной структуре невозможна полная компенсация потоков $S_{x}$ в вакууме и в структуре.

Рассмотрим симметричную структуру с двумя пленками толщины $t$ на каждой стороне диэлектрической пластины. В плоскости $z=0$ находится электрическая стенка. Трансформируя нормированный импеданс к поверхности пластины, имеем

$$
\rho_{i n}(d / 2)=i \rho \tan \left((d / 2) \sqrt{k_{0}^{2} \tilde{\varepsilon}-k_{x}^{2}}\right)
$$

Трансформируя этот импеданс к поверхности металлической пленки, имеем

$$
\rho_{i n}(t+d / 2)=\rho_{m} \frac{\rho_{i n}(d / 2)+i \rho_{m} \tan \left(t \sqrt{k_{0}^{2} \varepsilon-k_{x}^{2}}\right)}{\rho_{m}+i \rho_{i n}(d / 2) \tan \left(t \sqrt{k_{0}^{2} \varepsilon-k_{x}^{2}}\right)} .
$$

Приравнивая этот импеданс импедансу ПП в вакууме, получаем ДУ $\rho_{0}=\rho_{i n}(t+d / 2)$. Учитывая, что введенные импедансы, нормированные к импедансу $\left(\mu_{0} / \varepsilon_{0}\right)^{1 / 2}$, имеют вид $\rho_{0}=\sqrt{1-n^{2}}, \rho_{m}=\sqrt{\varepsilon-n^{2}} / \varepsilon$ и $\rho=\sqrt{\tilde{\varepsilon}-n^{2}} / \tilde{\varepsilon}$, указанное ДУ можно записать в виде $n=\sqrt{1-\rho_{i n}^{2}}$, где входной импеданс определяется уравнением (5). Нас будут интересовать не слишком медленные ПП, для которых $k_{0}^{2}<k_{x}^{2}<k_{0}^{2} \tilde{\varepsilon}$, $\rho>0, \rho_{m}=i\left|\rho_{m}\right|, \rho_{0}=-i \sqrt{n^{2}-1}$, поэтому обозначим $d \sqrt{k_{0}^{2} \tilde{\varepsilon}-k_{x}^{2}} / 2=\theta_{0}, t \sqrt{k_{x}^{2}-k_{0}^{2} \varepsilon}=2 \theta$. Тогда

$\rho_{\text {in }}(t+d / 2)=i\left|\rho_{m}\right| \frac{\rho \tan \left(\theta_{0}\right)+\left|\rho_{m}\right| \tanh (2 \theta)}{\left|\rho_{m}\right|+\rho \tan \left(\theta_{0}\right) \tanh (2 \theta)}=i \eta\left(k_{0}, k_{x}\right)$.

В работах [6-8] показано, что в диссипативных структуpax обратный ПП имеет место, если входной импеданс типа (5) емкостной на точном решении ДУ. Для (5) это условие имеет вид

$$
\left|\rho_{m}\right| / \tanh (2 \theta)<\rho \tan \left(\theta_{0}\right)<-\left|\rho_{m}\right| \tanh (2 \theta) .
$$

Однако для недиссипативной структуры оно неправомочно. Получим соответствующее условие существования обратного недиссипативного ПП. ДУ имеет вид $k_{x}=k_{0} \sqrt{1-\rho_{i n}^{2}\left(k_{0}, k_{x}\right)}$. Дифференцируя его по $k_{x}$, получаем

$$
\begin{gathered}
-\rho_{\text {in }}\left[\frac{\partial}{\partial k_{0}} \rho_{i n}\left(k_{0}, k_{x}\right) \frac{v_{g}}{c}+\frac{\partial}{\partial k_{x}} \rho_{i n}\left(k_{0}, k_{x}\right)\right] \\
=\left(n-n^{2} v_{g} / c\right) / k_{0} .
\end{gathered}
$$

Для обратного ПП $v_{g}<0$, и правая часть (8) положительная. Уравнение (8) позволяет определить точки, между которыми $v_{g}<0$, полагая $v_{g}=0$. Для очень малой ГС $v_{g} \ll c$, первым членом в квадратной скобке можно пренебречь, и имеем приближенное условие $\rho_{i n} \partial \rho_{i n} / \partial k_{x}<0$. Для импеданса (7), индуктивного при малой толщине, это означает возрастание индуктивной части $\eta$ с ростом замедления. Для ДУ (1) имеем

$$
\rho_{\text {in }}=-2 i \theta /\left(k_{0} t \varepsilon\right) \tanh (\theta)
$$

или $\rho_{i n}=-i \sqrt{k_{x}^{2} / k_{0}^{2}-1}$. В первом случае при $v_{g}=0$ из (8) получаем соотношения (2)-(4), а во втором случае (8) является тождеством. Равенство $v_{g}=0$ для (8) и (7) дает условие типа (2). Оно достаточно громоздкое и требует численного решения совместно с ДУ. Если его разрешить относительно $k_{0}$ или $k_{x}$ и подставить результат в ДУ, то все равно необходимо численно определять корни.

В случае тонкой пленки в вакууме ее можно описать поверхностной проводимостью

$$
\sigma=i \omega \varepsilon_{0}(\varepsilon(\omega)-1) t
$$

вне зависимости от того, проводящий ее материал или нет (в последнем случае имеет место ток поляризации). Для металлической пленки при $\varepsilon(\omega)<0$, а также на сверхнизких частотах, когда ДП большая по величине и мнимая отрицательная, поверхностная проводимость индуктивная. Однако выше частоты ППР она становится емкостной, что и определяет высокочастотные обратные ветви рис. 2, 3. Поверхностную проводимость можно вводить, когда толщина существенно меньше глубины проникновения $\delta$ и длины волны в пленке. В случае отрицательной ДП

$$
k_{z}=\sqrt{k_{0}^{2} \varepsilon-k_{z}^{2}}=i k_{0} \sqrt{|\varepsilon|+n^{2}}
$$

и при $|\varepsilon| \approx 1$ имеем

$$
\delta=\lambda /\left(2 \pi \sqrt{1+n^{2}}\right)
$$

поэтому для замедлений порядка нескольких единиц толщина до нескольких десятков нанометров и меньше удовлетворяет этому критерию.

Для двумерных материалов, таких как графен, используют скалярную проводимость на основе подходов метода динамических функций Грина, формул типа КубоГринвуда [12-18] или тензорную проводимость с учетом пространственной дисперсии, например в приближении 
времени релаксации (ПВР) или Бхатнагара-ГроссаКрука (БГК) [14]. ДУ Е-ПП для скалярной проводимости для пленки на обеих сторонах диэлектрической пластины получается наиболее просто:

$$
k_{x}=k_{0} \sqrt{1-\rho_{i n}^{2}\left(k_{0}, k_{x}\right)},
$$

где

$$
\begin{gathered}
\rho_{i n}=\sigma Z_{0}+2 i \tilde{\theta} \tan (\tilde{\theta}) /\left(k_{0} \tilde{\varepsilon} d\right), \\
\tilde{\theta}=d \sqrt{k_{0}^{2} \tilde{\varepsilon}-k_{x}^{2}} / 2 .
\end{gathered}
$$

В случае тензорной проводимости имеет место пространственная дисперсия (ПД), вид ДУ зависит от угла распространения ПП, т.е. от $k_{x}$ и $k_{y}$. В общем случае волны будут гибридными, т.е. представленными связанными Е-ПП и Н-ПП. Удобно классифицировать такие ПП относительно нормальной координаты $z$. Тогда для ПП с электрической стенкой в области $z=0$ внутри диэлектрического слоя имеем

$$
\begin{aligned}
& E_{z}=E \cosh \left(k_{z} z\right) \exp \left(-i k_{y} x-i k_{y} y\right), \\
& H_{z}=H \sinh \left(k_{z} z\right) \exp \left(-i k_{y} x-i k_{y} y\right) .
\end{aligned}
$$

Здесь $i k_{z}-$ продольная компонента волнового вектора, $k_{z}=\sqrt{k_{x}^{2}+k_{y}^{2}-k_{0}^{2} \tilde{\varepsilon}}$. Вне диэлектрического слоя

$$
\begin{aligned}
& E_{z}(z)=E_{0} \exp \left(-i k_{x} x-i k_{y} y-k_{z 0}|z|\right), \\
& H_{z}(z)=H_{0} \exp \left(-i k x-i k_{y} y-k_{z 0}|z|\right),
\end{aligned}
$$

где $k_{z 0}=\sqrt{k_{x}^{2}+k_{y}^{2}-k_{0}^{2}}-$ продольная компоненты в вакууме. Выражая все оставшиеся поперечные компоненты полей через продольные с помощью уравнений Максвелла и налагая при $z=d / 2$ граничные условия $E_{x}(d / 2+0)=E_{x}(d / 2-0), \quad E_{y}(d / 2+0)=$ $=E_{y}(d / 2-0), \quad J_{s x}=\sigma_{x x} E_{x}+\sigma_{x y} E_{y} \quad$ и $\quad J_{s y}=\sigma_{y x} E_{x}+$ $+\sigma_{y y} E_{y}, \quad$ где $\quad J_{s x}=H_{y}(d / 2-0)-H_{y}(d / 2+0)$, $J_{s y}=H_{x}(d / 2+0)-H_{x}(d / 2=0)$, получим систему четырех однородных алгебраических уравнений, равенство нулю определителя которой и есть ДУ:

$$
\operatorname{det}(A)=0
$$

Матрица $A$ имеет следующие коэффициенты:

$$
\begin{gathered}
A_{11}=\frac{i k_{x} k_{z 0}}{k_{0}^{2}+k_{z 0}^{2}}, \quad A_{12}=\frac{-k_{y} k_{0}}{k_{0}^{2}+k_{z 0}^{2}} \\
A_{13}=\frac{i k_{x} k_{z} \sinh \left(k_{z} d / 2\right)}{k_{0}^{2} \tilde{\varepsilon}+k_{z}^{2}}, \quad A_{14}=\frac{k_{y} k_{0} \sinh \left(k_{z} d / 2\right)}{k_{c}^{2}} \\
A_{21}=\frac{i k_{y} k_{z 0}}{k_{0}^{2}+k_{z 0}^{2}}, \quad A_{22}=\frac{k_{x} k_{0}}{k_{0}^{2}+k_{z 0}^{2}}, \\
A_{23}=\frac{i k_{y} k_{z} \sinh \left(k_{z} d / 2\right)}{k_{0}^{2} \tilde{\varepsilon}+k_{z}^{2}}, \quad A_{24}=-\frac{k_{x} k_{0} \sinh \left(k_{z} d / 2\right)}{k_{0}^{2} \tilde{\varepsilon}+k_{z}^{2}} \\
A_{31}=\frac{i k_{0 z}\left(\xi_{x x} k_{x}+\xi_{x y} k_{y}\right)-k_{0} k_{x}}{k_{0}^{2}+k_{z 0}^{2}}
\end{gathered}
$$

$$
\begin{gathered}
A_{32}=\frac{k_{0}\left(\xi_{x y} k_{x}-\xi_{x x} k_{y}\right)+i k_{y} k_{0 z}}{k_{0}^{2}+k_{z 0}^{2}}, \\
A_{33}=\frac{k_{0} k_{x} \tilde{\varepsilon} \cosh \left(k_{z} d / 2\right)}{k_{0}^{2} \tilde{\varepsilon}+k_{z}^{2}}, \quad A_{34}=\frac{i k_{y} k_{z} \cosh \left(k_{z} d / 2\right)}{k_{0}^{2} \tilde{\varepsilon}+k_{z}^{2}}, \\
A_{41}=\frac{i k_{0 z}\left(\xi_{x y} k_{x}+\xi_{y y} k_{y}\right)-k_{0} k_{y}}{k_{0}^{2}+k_{z 0}^{2}}, \\
A_{42}=\frac{k_{0}\left(\xi_{y y} k_{x}-\xi_{x y} k_{y}\right)-i k_{x} k_{0 z}}{k_{0}^{2}+k_{z 0}^{2}} \\
A_{43}=\frac{\tilde{\varepsilon} k_{0} k_{y} \cosh \left(k_{z} z\right)}{k_{0}^{2} \tilde{\varepsilon}}, \quad A_{44}=-\frac{i k_{x} k_{z} \cosh \left(k_{z} z\right)}{k_{0}^{2} \tilde{\varepsilon}+k_{z}^{2}} .
\end{gathered}
$$

Здесь использованы соотношения симметрии ОнсагераКазимира $\sigma_{x y}=\sigma_{y x}$ и обозначены нормированные компоненты тензора проводимости $\xi_{x x}=Z_{0} \sigma_{x x}, \xi_{x y}=Z_{0} \sigma_{x y}$, $\xi_{y y}=Z_{0} \sigma_{y y}$. Рассмотрим частный случай $k_{y}=0$. Тогда

$$
\begin{gathered}
\left|\begin{array}{cccc}
A_{11} & 0 & A_{13} & 0 \\
0 & A_{22} & 0 & A_{24} \\
A_{31} & A_{32} & A_{33} & 0 \\
A_{41} & A_{42} & 0 & A_{44}
\end{array}\right| A_{11}\left|\begin{array}{ccc}
A_{22} & 0 & A_{24} \\
A_{32} & A_{33} & 0 \\
A_{42} & 0 & A_{44}
\end{array}\right| \\
+A_{13}\left|\begin{array}{ccc}
0 & A_{22} & A_{24} \\
A_{31} & A_{32} & 0 \\
A_{41} & A_{42} & A_{44}
\end{array}\right|=0 .
\end{gathered}
$$

Имеем более простое дисперсионное уравнение

$$
\begin{aligned}
\operatorname{det}(A)= & A_{11}\left(A_{22} A_{33} A_{44}-A_{42} A_{33} A_{24}\right) \\
& +A_{13}\left(A_{31} A_{42} A_{24}-A_{41} A_{32} A_{24}-A_{31} A_{22} A_{44}\right)=0 .
\end{aligned}
$$

В нем имеются упрощения:

$$
\begin{gathered}
A_{31}=\left(i k_{0 z} \xi_{x x}-k_{0}\right) /\left(k_{0}^{2}+k_{z 0}^{2}\right), \\
A_{32}=\xi_{x y} k_{x} k_{0} /\left(k_{0}^{2}+k_{z 0}^{2}\right), \\
A_{41}=i \xi_{x y} k_{x} k_{0 z} /\left(k_{0}^{2}+k_{z 0}^{2}\right), \\
A_{42}=\left(\xi_{y y} k_{x} k_{0}-i k_{0 z} k_{x}\right) /\left(k_{0}^{2}+k_{z 0}^{2}\right) .
\end{gathered}
$$

Пусть тензор проводимости приведен к главным осям (диагонализирован) в рассматриваемой системе координат. Тогда $A_{32}=A_{41}=0$ и

$$
\operatorname{det}(A)=\left(A_{11} A_{33}-A_{13} A_{31}\right)\left(A_{22} A_{44}-A_{42} A_{24}\right)=0 .
$$

Это ДУ распадается на два - для Е-НН и Н-ПП:

$$
A_{11} A_{33}-A_{13} A_{31}=0, \quad A_{22} A_{44}-A_{42} A_{24}=0 .
$$

Для первого имеем

$$
k_{x}=k_{z} \tanh \left(k_{z} d / 2\right)\left(i k_{0} \tilde{\varepsilon} \xi_{x x}-k_{0}^{2} \tilde{\varepsilon} / k_{0 z}\right) .
$$

Для второго

$$
-k_{z}=\left(i \xi_{y y} k_{0}+k_{z 0}\right) \tanh \left(k_{z} d / 2\right) .
$$


Если $\xi_{y y}=0$ (отсутствие пленки), это уравнение имеет вид

$$
-\sqrt{\left(k_{x}^{2}-k_{0}^{2} \tilde{\varepsilon}\right) /\left(k_{x}^{2}-k_{0}^{2}\right)}=\tanh \left(k_{z} d / 2\right) .
$$

Это ДУ магнитного симметричного ПП вдоль слоя диэлектрика. Возводя уравнение (10) в квадрат, приведем его к форме

$$
k_{x}=\sqrt{k_{0}^{2} \tilde{\varepsilon}-\left(i \xi_{y y} k_{0}+k_{z 0}\right)^{2} \tanh ^{2}\left(k_{z} d / 2\right)} .
$$

Оно, как и (1), неявное относительно $k_{x}$. Для очень толстой слабо диссипативной пластины $\tanh ^{2}\left(k_{z} d / 2\right)=1$, и $k_{x}$ находится из биквадратного уравнения

$$
k_{x}^{2}=k_{0}^{2} \tilde{\varepsilon}-\left(i \xi_{y y} k_{0}+\sqrt{k_{x}^{2}-k_{0}^{2}}\right)^{2},
$$

а при отсутствии пленки решения нет: магнитный ПП не может существовать над диэлектрическим полупространством. Это соответствует тому, что для падающей плоской волны не существует угла отсутствия отражения (угла Брюстера). Аналогично можно рассмотреть ДУ (9).

Заметим, что все приведенные выше окончательные результаты для магнитной стенки в плоскости $z=0$ получаются заменой $\tanh (\theta) \rightarrow 1 / \tanh (\theta)$ и $\tanh (\tilde{\theta}) \rightarrow 1 / \tanh (\tilde{\theta})$. Все ДУ для Е-ПП, в которые входят нормированные входные сопротивления $\rho_{i n}$, формально получаются заменой последних на проводимости: $\rho_{i n} \rightarrow 1 / \rho_{\text {in }}$. При этом указанные величины следует вычислять для соответствующих типов волн.

Рассмотрим простейшие ДУ с металлической пленкой. Для Е-ПП вдоль металлической пленки в бесконечном диэлектрическом пространстве ДУ имеет вид

$$
k_{x}=k_{0} \sqrt{\tilde{\varepsilon}-4 \tilde{\varepsilon}^{2} /\left(\sigma Z_{0}\right)^{2}},
$$

где $\sigma Z_{0}=i k_{0} t(\varepsilon(\omega)-\tilde{\varepsilon})$. Это соотношение следует из того, что дополнительная плотность тока поляризации при расположении в диэлектрике

$$
\mathbf{J}_{p}=i \omega \varepsilon_{0}(\varepsilon(\omega)-\tilde{\varepsilon}) \mathbf{E} .
$$

Максимальное замедление Е-ПП растет с уменышением $\omega_{c}$ и $\tilde{\varepsilon}^{\prime \prime}$, а также при приближении $\tilde{\varepsilon}^{\prime}$ к $\varepsilon_{L}$. Технологически тонкие пленки могут быть выполнены в диэлектрике (на границе двух диэлектрических слоев) или на диэлектрической подожке. Поэтому учет диэлектрических слоев и подложек необходим, что приводит к более сложным ДУ. Простые явные уравнения типа (12), позволяющие точно определять дисперсию и ГС, наиболее удобны для решения вопроса о том, связана ли отрицательная ГС с с обратными волнами, поскольку последние определяются знаком $\sigma^{\prime \prime}$. Для активной пленки $\left(\sigma^{\prime}<0\right)$ волна вытекает (с конечного участка излучается). Для графена этому соответствует $\omega_{c}<0$. Вытекающий Е-ПП обратный, если $\sigma^{\prime} \sigma^{\prime \prime}<0$, т. е. для индуктивной проводимости пленки. В случае пленки на толстой диэлектрической подложке берем для нее модель диэлектрического полупространства с ДП $\tilde{\varepsilon}$. Дисперсию ДП и диссипацию не учитываем. Тогда ДУ имеет вид

$$
y_{0}^{(e, h)}=y_{d}^{(e, h)}+\sigma Z_{0} .
$$

Здесь $\quad y_{d}^{e}=k_{z d} /\left(k_{0} \tilde{\varepsilon}\right), \quad y_{d}^{h}=k_{0} / k_{z d}, \quad y_{0}^{e}=-i k_{z 0} / k_{0}$, $y_{0}^{h}=i k_{0} / k_{z 0}$. Если рассмотреть диэлектрическую пластину толщины $d$ с ДП $\tilde{\varepsilon}$, обложенную с обеих сторон проводящими пленками, то получаем ДУ

$$
y_{0}^{(e, h)}=\left[i \tan \left(k_{z d} d / 2\right)\right]^{ \pm 1} y_{d}^{(e, h)}+\sigma Z_{0} .
$$

Верхний знак соответствует моде с магнитной стенкой, а нижний - с электрической, $k_{z d}=\sqrt{k_{0}^{2} \tilde{\varepsilon}-k_{x}^{2}}=-i k_{z}$, $y_{d}^{e}=k_{0} \tilde{\varepsilon} / k_{z d}, y_{d}^{h}=k_{0} / k_{z d}$. Рассмотрим случай очень медленного ПП, $k_{x}^{\prime}>k_{0} \sqrt{\tilde{\varepsilon}}$, при условии малой диссипации. Тогда $k_{z d}=-i k_{z} \approx-i k_{x}, y_{d}^{e} \approx i\left|y_{d}^{e}\right|, y_{d}^{h} \approx i\left|y_{d}^{h}\right|$, $\tan \left(k_{z d} d / 2\right)=-i \tanh \left(k_{z} d / 2\right)$, поэтому к проводимости пленки добавляется индуктивная проводимость в случае Е-ПП и Н-ПП. Более сильное увеличение индуктивной проводимости имеет место для моды с электрической стенкой, поскольку при малой толщине $d$ и не очень больших замедлениях гиперболический тангенс мал. При $d \rightarrow \infty$ следует положить $i \tan \left(k_{z d} d / 2\right)=1$, и из (13) следует ДУ для пленки на полупространстве. При $d \rightarrow 0$ имеем $y_{0}^{(e, h)}=\sigma Z_{0}$. Это ДУ совпадает с ранее приведенным, если учесть, что полную проводимость такой двусторонней пленки следует удвоить.

Рассмотрим пленку в центре диэлектрической пластины. Для получения ДУ трансформируем половинную проводимость к границе с вакуумом:

$$
y_{i n}^{(e, h)}=y_{d}^{(e, h)} \frac{\sigma Z_{0} / 2+i \tan \left(k_{z d} d / 2\right) y_{d}^{(e, h)}}{y_{d}^{(e, h)}+i \tan \left(k_{z d} d / 2\right) \sigma Z_{0} / 2} .
$$

Тогда ДУ имеет вид $y_{0}^{(e, h)}=y_{i n}^{(e, h)}$. Поскольку $\sigma$ имеет действительную часть, изменение знака реактивной части в (21) возможно. Рассмотрим случай не очень медленного ПП: $k_{0}<k_{x}^{\prime}<k_{0} \sqrt{\varepsilon_{d}}$. Для него $k_{z d}$ и проводимости $y_{d}^{(e, h)}$ почти действительные, поэтому для не очень толстой пластины для ПП с магнитной стенкой добавляется индуктивный импеданс, а для ПП с электрической стенкой - емкостной импеданс. В случае толстой слабо диссипативной подложки тангенс может менять знак и изменяться в широких пределах с изменением частоты, а поведение входной проводимости (14) и дисперсии ПП может быть сложным. Следует заметить, что при слабой диссипации в пластине тангенс не достигает бесконечных значений, но может быть большим по модулю.

В случае графена динамическая проводимость без учета ПД комплексная, $\sigma(\omega)=\sigma_{\text {intra }}(\omega)+\sigma_{\text {inter }}(\omega)$, причем в большей части диапазона индуктивная. Для межзонной проводимости $\sigma_{\text {inter }}$ температурной зависимостью по 
сравнению с химическим потенциалом можно пренебречь (при $\left.k_{B} T \ll\left|\mu_{c}\right|\right)$, и тогда имеем [12-18]

$$
\begin{gathered}
\sigma_{\text {intra }}\left(\omega, \mu, \omega_{c}, T\right)=\frac{-i e^{2} k_{B} T}{\pi \hbar^{2}\left(\omega-i \omega_{c}\right)} \varphi\left(\mu_{c}, T\right) \\
=\frac{\sigma_{0 \text { intra }}}{1+i \omega / \omega_{c}}, \\
\sigma_{\text {inter }}\left(\omega, \mu, \omega_{c}, 0\right)=\frac{-i e^{2}}{4 \pi \hbar} \ln \left(\frac{2\left|\mu_{c}-\left(\omega-i \omega_{c}\right) \hbar\right|}{2\left|\mu_{c}+\left(\omega-i \omega_{c}\right) \hbar\right|}\right), \\
\varphi\left(\mu_{c}, T\right)=\ln \left(2+2 \cosh \left(\frac{\mu_{c}}{k_{B} T}\right)\right) .
\end{gathered}
$$

Хорошим приближением является модель типа Друде $\sigma \approx e^{2} /(4 \hbar)+\sigma_{0}\left(1+i \omega / \omega_{c}\right)$. Однако в узком диапазоне частот $\sigma$ может быть емкостной. Для учета ПД в случае ПП вдоль главной оси графена в приближении БГК следует использовать компоненту [14]

$$
\begin{aligned}
\sigma_{x x}(\omega)= & \sigma_{\text {intra }}(\omega)\left[1+\frac{v_{F}^{2}}{4\left(\omega-i \omega_{c}\right)^{2}}\left(3-\frac{2 i}{\omega / \omega_{c}}\right) k_{x}^{2}\right] \\
& +\sigma_{\text {inter }}(\omega) .
\end{aligned}
$$

Для ПВР коэффициенты несколько отличаются [14]. Здесь $v_{F} \approx c / 300$. Дисперсию Е-ПП вдоль пленки в вакууме получаем, трансформируя проводимость вакуума за пленкой $y_{0}^{(e, h)}=1 / \rho_{0}^{(e, h)}$ к проводимости вакуума перед пленкой. Пленка шунтирует проводимость, т.е. $y_{i n}^{(e, h)}=\sigma Z_{0}-y_{0}^{(e, h)}$. Знак справа у проводимости отрицательный, поскольку волна втекает справа в диссипативную пленку. ДУ имеет вид $y_{0}^{(e, h)}=y_{i n}^{(e, h)}=\sigma Z_{0}-y_{0}^{(e, h)}$ или $k_{x}=k_{0} \sqrt{1-\left(\sigma Z_{0} / 2\right)^{\mp 2}}$. Здесь верхний знак соответствует Е-ПП, а нижний - Н-ПП. Эти же уравнения получаются путем сшивания с учетом скачка магнитного поля. Для медленного Е-ПП нормированная проводимость должна быть малой и сильно реактивной. Для медленного Н-ПП нормированная проводимость должна быть большой и сильно реактивной. На рис. 4 представлена дисперсия и диссипация Е-ПП. Видно, что в узких полосах потери отрицательные. Все дисперсионные кривые рис. 1-4 для удобства построены так, что $k_{x}^{\prime}>0$. Поэтому отрицательные потери соответствуют обратному плазмону.

Потери приводят к возможности распространения плазмон-поляритонов в запрещенных зонах, при этом аномальная отрицательная дисперсия не означает наличие обратных плазмонов [6-10]. Итерационное решение ДУ (1) с диссипацией показывает, что в области плазмонного резонанса нет замедленных обратных ПП рис. 3. Обратные замедленные ПП имеют место в области $\varepsilon^{\prime}>0$. В области $-1<\varepsilon^{\prime}<0$ при малой толщине $n^{\prime} \approx 1$ потери отрицательные и очень малые (сливающиеся кривые 1,2, 3 на рис. 2.). Такой обратный быстрый плазмон не очень интересен. Причина отсутствия замедленных обратных ПП в том, что имеет место
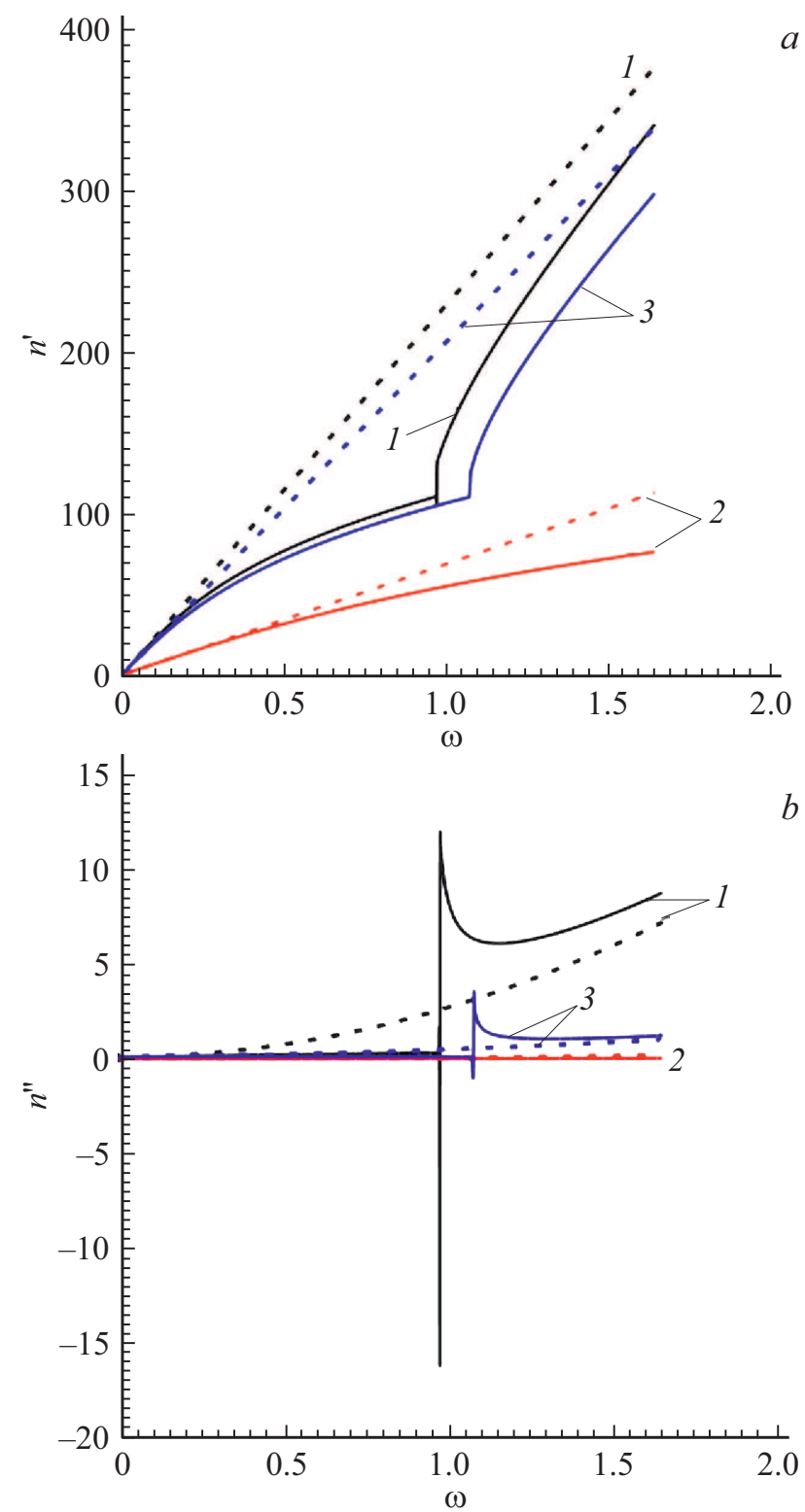

Рис. 4. Замедление $n^{\prime}(a)$ и потери $n^{\prime \prime}(b)$ Е-ПП в графеновой пленке при $T=300 \mathrm{~K}$ в зависимости от круговой частоты $(\mathrm{eV}): \mu_{c}=0.3 \mathrm{eV}, \tilde{\varepsilon}=1$ (кривые 1 ); $\mu_{c}=1 \mathrm{eV}, \tilde{\varepsilon}=1$ (2); $\mu_{c}=1 \mathrm{eV}, \tilde{\varepsilon}=3-0.003 i$ (3). Штриховые кривые - без учета ПД, сплошные кривые - с учетом ПД.

втекание энергии из вакуума в пленку. Втекание сопровождается диссипацией энергии в пленке. Направление вектора Пойнтинга в пленке определяется не только знаком $\varepsilon^{\prime}$, но и величинами $k_{x}^{\prime}$ и $k_{x}^{\prime \prime}$. Достаточно громоздкие вычисления показывают, что условие $k_{x}^{\prime} k_{x}^{\prime \prime}<0$ в области $\varepsilon^{\prime}<-1$ при решении ДУ не выполняется. Однако в других областях для различных типов ПП возможны случаи обратных плазмонов при нормальной дисперсии, а также прямых плазмонов при аномальной дисперсии [6]. На рис. 4 приведены дисперсионные кривые медленных в области $\varepsilon^{\prime}-1$ антисимметричных ПП (рис. 1, 2, 3). 
Получим условия существования обратных Е-ПП в тонких пленках на подложке. Для несимметричной структуры с двумя разными пленками снизу от структуры берем нормированную проводимость вакуума с отрицательным знаком: $-y_{0}^{(e, h)}$. В указанную проводимость входит величина $k_{z 0}= \pm \sqrt{k_{0}^{2}-k_{x}^{2}}$, знак которой определяется втеканием или вытеканием [10]. Поскольку структура диссипативная, а ПП медленный, следует задать условие втекания. У указанной проводимости следует добавить нормированную проводимость пленки на нижней стороне пластины: $\sigma Z_{0}-y_{0}^{(e, h)}$. Это нормированная нагрузочная проводимость, которую следует трансформировать к верхней поверхности подложки по формуле типа (14), в которой используем $\tan \left(d \sqrt{k_{0}^{2} \tilde{\varepsilon}-k_{x}^{2}}\right)$. Обозначим эту проводимость $y_{t r}^{(e, h)}$. К ней следует добавить нормированную проводимость второй пленки $\sigma_{+}: y_{i n}^{(e, h)}=\sigma_{+}+y_{t r}^{(e, h)}$. Это входная проводимость на верхней стороне структуры. ДУ имеет вид $y_{0}^{(e, h)}=y_{i n}^{(e, h)} \sigma_{+}+y_{t r}^{(e, h)}$ (это опять условие втекания). Условие существования обратного ПП состоит в том, что $y_{i n}^{(e, h)}$ является емкостной. Для тонких металлических пленок в области плазмоники двумерные (поверхностные) проводимости индуктивные. Поэтому диэлектрическая пластина должна быть достаточно толстой. Удобно рассмотреть симметричную структуру $\left(\sigma_{ \pm}=\sigma\right)$. Имеем

$$
y_{i n}^{(e, h)}=\sigma Z_{0}+y_{d}^{(e, h)}\left[i \tan \left(d \sqrt{k_{0}^{2} \tilde{\varepsilon}-k_{x}^{2}} / 2\right)\right]^{ \pm 1} .
$$

В (15) знак „,““ соответствует магнитной стенке, а знак, „-“ - электрической стенке в плоскости $z=0$. Имеем $n^{e}=\sqrt{1-\left(y_{i n}^{e}\right)^{-2}}, n^{h}=\sqrt{1-\left(y_{i n}^{e}\right)^{2}}$. Пусть диссипация слабая. Тогда реальные части (15) существенно меньше мнимых, т.е. входные проводимости сильно реактивные. Рассмотрим условие существенного замедления ПП. Имеем $n^{\prime} e \gg 1$, если $\operatorname{Re}\left(y_{i n}^{e}\right) \ll \operatorname{Im}\left|\left(e_{i n}^{e}\right)\right| \ll 1$. С другой стороны, $n^{\prime h} \gg 1$, если $\operatorname{Re}\left(y_{i n}^{h}\right) \ll \operatorname{Im}\left|\left(y_{i n}^{h}\right)\right|$ и $1 \ll \operatorname{Im}\left|\left(y_{i n}^{h}\right)\right|$. Если пластина толстая, тангенс в (15) имеет отрицательный знак, причем емкостная проводимость правого члена немного превышает индуктивную проводимость пленки, Е-ПП медленный и обратный. Для Н-ПП указанное превышение имеет место при меньшей толщине пленки, и тогда Н-ПП обратный. Следует иметь в виду, что все рассмотрение проведено для замедлений $n^{\prime}(e, h)<\sqrt{\tilde{\varepsilon}}$, что предполагает большую ДП пластины. Рассмотрим случай очень больших замедлений $n^{\prime}(e, h)>\sqrt{\tilde{\varepsilon}}$. Тогда

$$
y_{i n}^{(e, h)}=\sigma Z_{0} \pm i\left|y_{d}^{(e, h)}\right|[\tanh (\tilde{\theta})]^{ \pm 1},
$$

знак плюс соответствует электрическому, минус магнитному ПП, $\left|y_{d}^{e}\right|=\tilde{\varepsilon} / \sqrt{n^{2}-\tilde{\varepsilon}},\left|y_{d}^{h}\right|=\sqrt{n^{2}-\tilde{\varepsilon}}$. Такой Е-ПП не может быть обратным, а Н-ПП будет обратным, если

$$
\operatorname{Im}\left(\sigma Z_{0}\right)<\operatorname{Re}\left(\sqrt{n^{2}-\tilde{\varepsilon}}[\tanh (\tilde{\theta})]^{ \pm 1}\right) .
$$

В случае очень большого замедления это условие можно записать так: $\operatorname{Im}\left(\sigma Z_{0}\right)<n^{\prime h}$.

\section{Заключение}

В заключение отметим, что диссипацию можно не учитывать, если $\omega_{c}$ существенно меньше минимальной частоты рассматриваемого диапазона, а замедление не слишком велико. С ростом замедления потери ПП растут. Существенное снижение частоты столкновений возможно при сверхнизких температурах, а при комнатной учет диссипации важен. Он требует поиска комплексных корней $k_{x}=k_{x}^{\prime}-i k_{x}^{\prime \prime}$, что позволяет определить область обратного ПП как $k_{x}^{\prime} k_{x}^{\prime \prime}<0$. Если $n^{\prime} \approx 1$, то ПП идет почти со скоростью света, и его потери очень малы. Соответствующее ДУ необходимо решать с очень высокой точностью, чтобы определить знак $k_{x}^{\prime \prime}$. Более точный результат в этом случае дает вычисление $\left\langle S_{x}\right\rangle$.

Для преодоления влияния диссипации в ряде работ предлагается использовать активные слои или структуры (спазеры) [31] и сверхнизкие температуры. Интересны активные полупроводниковые структуры и накачанные листы графена. Использованный подход позволяет это учитывать чисто формально, используя отрицательную частоту столкновений. Следует отметить, что если все слои структуры имеют отрицательные потери, то условия для обратных ПП изменяются: $k_{x}^{\prime} k_{x}^{\prime \prime}>0$, т.е. такая структура является усиливающей. При этом волна становится вытекающей: энергия из структуры вытекает в вакуум (излучается). Использование тонких диэлектрических пленок с большой ДП и малыми потерями также позволяет снизить потери ПП, при этом такая пленка описывается емкостной поверхностной проводимостью. Важно только выполнение условия двумерности, иначе толстую пленку следует описывать как объемную структуру. В случае замагниченных тонких металлических пленок их ДП становится тензорной, как и связанная с ней проводимость. Аналогично тонкие ферритовые пленки можно рассматривать как поверхностные магнитные токи с плотностью $\mathbf{J}_{s}^{m}=i \omega \mu_{0}(\hat{\mu}-\hat{I}) \mathbf{H}$, позволяющей упростить ряд ДУ теории магнитостатических волн (МСВ) для очень тонких пленок. В ряде работ, например в [32], получен загиб дисперсионной кривой диссипативных поверхностных MCB в запрещенную область, который трактуется как диссипативные обратные поверхностные МСВ только на основе характера дисперсии, что, как показано в настоящей работе, делать нельзя. Введение двумерных (поверхностных) проводимостей тонких пленок требует решения квантовых задач для соответствующих им квантовых ям с определением числа мод проводимости, динамической проводимости с учетом релаксации (рассеяния на фононах). Определение такой проводимости, зависящей от толщины, представляет собой отдельную проблему, а использование объемной ДП модели ДрудеЛоренца является приближением. Также приближением 
является использование проводимости графена при его расположении на подложке: указанную проводимость следует получать с учетом влияния подложки.

В работе получены ДУ и дисперсионные характеристики ПП для различных структур с тонкими пленками, установлены условия существования прямых и обратных, быстрых и медленных, а также втекающих и вытекающих ПП. Рассмотрены диссипативные и недиссипативные ПП. Найдены их максимальные замедления и потери. Показано, что для диссипативных ПП критерием существования обратных волн является емкостной входной импеданс структуры со стороны вакуума. Рассмотренные волноведущие структуры перспективны в оптическом и терагерцевом диапазонах.

\section{Список литературы}

[1] Economou E.N. // Phys. Rew. 1969. V. 182. N 2. P. 539-54.

[2] Tournoisa P., Laude V. // Opt. Commun. 1997. V. 137. P. 41-45.

[3] Liu Y.M., Pile D.F.P., Liu Z.W. et al. // Proc. SPIE. 2006. V. 6323. P. $63231 \mathrm{M}$.

[4] Федянин Д.Ю., Арсенин А.В., Лейман В.Г., Гладун А.Д. // Квант. электрон. 2009. V. 39. № 8. Р. 745-760.

[5] Зуев В.С., Зуева Г.Я. // Опт. и спектр. 2008. Т. 105. C. $852-859$.

[6] Давидович М.В. // Квант. электрон. 2017. Т. 47. № 6. C. $567-579$.

[7] Давидович М.В. // Письма в ЖТФ. 2017. Т. 43. № 22. C. $55-62$.

[8] Давидович М.В., Мещанов В.П. // Антенны. 2017. № 8(240). C. 3-16.

[9] Давидович М.В. Втекающие и вытекающие несобственные моды - анализ диссипативных дисперсионных уравнений и волна Ценнека. Саратов: изд-во Сарат. ун-та, 2014. 106 с.

[10] Давидович М.В. // Радиотехн. и электрон. 2018. Т. 63. № 6. C. $499-506$.

[11] Fedyanin D.Yu., Arsenin A.V., Leiman V.G., Gladun A.D. // J. Opt. 2010. V. 12. P. 015002(7).

[12] Mikhailov S.A., Ziegler K. // Phys. Rev. Lett. 2007. V. 99(1). P. 016803(4).

[13] Hanson G.W. // J. Appl. Phys. 2008. V. 103. P. 064302(8).

[14] Lovat G., Hanson G.W., Araneo R., Burghignoli P. // Phys. Rev. B. 2013. V. 87. P. 115429(11).

[15] Falkovsky L.A., Pershoguba S.S. // Phys. Rev. B. 2007. V. 76. P. $153410(4)$.

[16] Falkovsky L.A., Varlamov A.A. // Eur. Phys. J. B. 2007. V. 56. P. 281-284

[17] Фальковский Л.А. // УФН. 2008. Т. 178. № 9. С. 923-934.

[18] Фальковский Л.А. // ЖЭТФ. 2012. Т. 142. № 3. С. 560-573.

[19] Ju L., Geng B., Horng J. et al. // Nature Nanotechnology. 2011. V. 6. P. 630-634.

[20] Буслаев П.И., Иори И.В., Шадривов И.В., Белов П.А., Кивщарь Ю.С. // Письма в ЖЭТФ. 2013. Т. 97. № 9. C. $619-623$.

[21] Karimi F., Knezevic I. // Phys. Rev. B. 2017. V. 96. P. $125417(12)$.

[22] Jablan M., Soljacic M., Buljan H. // Proc. IEEE. 2013. V. 101. N 7. P. $1689-1704$.
[23] Xiao S., Zhu X., Li B.-H., Mortensen N.A. // Front. Phys. 2016. V. 11(2). P. 117801(13).

[24] Давидович М.В. // ЖТФ. 2010. Т. 80. № 5. С. 40-44.

[25] Новотный Л., Хехт Б. Основы нанооптики. М.: Физматлит, 2009. $484 \mathrm{c.}$

[26] Raether H. Surface Plasmons on Smooth and Rough Surfaces and on Gratings. Berlin: Springer Verlag, Heidelberg, 1988. $135 \mathrm{p}$.

[27] Климов В.В. Наноплазмоника. М.: Физматлит, 2009. 480 с.

[28] Майер C.A. Плазмоника: Теория и приложения. М., Ижевск: Изд-во НИЦ „Регулярная и хаотическая динамика“, 2011. 396 с.

[29] Марков Г.Т., Чаплин А.Ф. Возбуждение электромагнитных волн. М.: Радио и связь, 1983. 296 с.

[30] Вайнштейн Л.А. Электромагнитные волны. М.: Радио и связь, 1988. $440 \mathrm{c.}$

[31] Виноградов А.П., Андрианов Е.С., Пухов К. и др. // УФН. 2012. T. 182. C. $1122-1130$.

[32] Келлер Ю.И., Макаров П.А., Шавров В.Г., Щеглов В.И. // Журн. радиоэлектроники. 2016. № 3. C. 7. [URL: http://jre.cplire.ru/jre/mar16/1/text.html] 